Article

\title{
Analysis of human breast milk microbiome and bacterial extracellular vesicles in healthy mothers
}

\author{
Su Yeong Kim ${ }^{1}$ and Dae Yong Yi ${ }^{1,2, *}$ \\ 1 Department of Pediatrics, Chung-Ang University Hospital, Seoul, 06973, South Korea; \\ oiya1024@hanmail.net (S.Y.K.) \\ 2 College of Medicine, Chung-Ang University, Seoul, 06911, South Korea \\ * Correspondence: meltemp2@hanmail.net; Tel.: +82-(0)2-6299-1480; Fax: +82-(0)2-6299-2064 (D.Y.Y.)
}

\begin{abstract}
The microbiota of human breast milk (HBM) contributes to infant gut colonization; however, whether bacterial extracellular vesicles (EVs) are present in HBM or might contribute to this process remains unknown. In the present study, we characterized the HBM microbiota of healthy Korean mothers and measured the key bacteria likely affecting infant gut colonization by analyzing both the microbiota and bacterial EVs. A total of $22 \mathrm{HBM}$ samples were collected from lactating mothers. The DNA of bacteria and bacteria-derived EVs was extracted from each sample. Gene analysis was performed using Illumina MiSeq. Firmicutes accounted for the largest portion among the phyla, followed by Proteobacteria, Bacteroides, and Actinobacteria in both bacteria and bacterial EV samples. At the genus level, Streptococcus (25.1\%) and Staphylococcus (10.7\%) were predominant in bacterial samples, whereas Bacteroides $(9.1 \%)$, Acinetobacter (6.9\%), and Lactobacillaceae(f) $(5.5 \%)$ were prevalent in bacterial EV samples. Several genera including Bifidobacterium were significantly positively correlated between the two samples. Our findings reveal the diverse bacterial communities in HBM of healthy lactating mothers and suggest the presence of key bacteria with metabolic activity in HBM and that EVs derived from these bacteria may contribute to the vertical transfer of gut microbiota from mother to infant.
\end{abstract}

Keywords: milk; human; microbiota; microbiome; extracellular vesicle; breastfeeding

\section{Introduction}

More than 100 trillion microbes dwell within the human gut and interact with the host in a symbiotic relationship to aid the development of the host immune system. The early acquisition of the gut microbiome during infancy affects the health throughout the human life. Several factors, such as the type of delivery, antibiotic therapy, environment of care, and nutritional exposure are assumed to influence the early colonization of the infant gut [1]. Diet plays a key role in the development of the newborn's immune system. Human breast milk (HBM) is not only the best source of nutrition for infants, but it is also known to contain immune components, such as secretory antibodies, immune cells, antimicrobial proteins (such as lactoferrin and lysozyme), cytokines, and human milk oligosaccharide [2]. Before the 2000s, the presence of bacteria in HBM was thought to indicate contamination or infection, but several researchers demonstrated that HBM contained commensal bacteria by using culture-dependent techniques [3,4]. The development of the non-culture sequencing technique has led to a more thorough investigation of the diversity of HBM microbiota [5]. Nowadays, it is recognized that HBM contains abundant commensal bacteria that can affect the colonization of the infant gut [6] .

Extracellular vesicles (EVs) are nanometer-sized membrane vesicles that contain various bioactive molecules such as transmembrane proteins, cytosolic proteins, nucleic acids, and lipids. It is widely accepted that all kinds of bacteria release EVs. Bacteria are classified into gram-positive $(\mathrm{G}+)$ and gram-negative (G-) bacteria based on membrane characteristics, where the G+ bacteria-derived EVs are usually called bacterial membrane vesicles and the G- bacteria-derived EVs 
are called outer membrane vesicles [7]. Bacteria-derived EVs can be detected in body fluids such as blood, urine, or stool, indicating that they can affect the host cells by directly activating host receptors, delivering various bioactive molecules, or integrating EVs into the host cells [8-10] . However, there has been no study on the analysis of bacteria-derived EVs in HBM until now.

In this context, the objective of this study was to characterize the HBM microbiota of healthy lactating mothers in Korea, and to measure the abundance of key bacteria that can affect the infant's gut colonization by analyzing both the microbiota and bacterial EVs using the culture independent technique.

\section{Materials and Methods}

\subsection{Subjects and Sample collection}

This study was conducted according to the guidelines proposed by the Declaration of Helsinki and approved by the Institutional Review Board (IRB) of Chung-Ang University Hospital, Seoul, South Korea (IRB No: 1810-004-309). Healthy lactating mothers who delivered a full-term baby within one week to four months were recruited for the present study (July 2017 to June 2018).

Samples of breast milk were collected from 22 mothers in sterile bags by manual or pump expression. Before milk expression, hands were washed and nipples were cleaned with sterile water. The first ten drops were discarded. The fresh samples were delivered to the laboratory within $24 \mathrm{~h}$ and then they were stored at $-80{ }^{\circ} \mathrm{C}$ until further processing. If the samples did not reach the laboratory within $24 \mathrm{~h}$, they were excluded. The bacterial diversity changes based on the lactation stage, especially the difference between the microbiomes of colostrum and mature HBM is well known [1]. As we tried to analyze the bacteria that infants actually consume while feeding on stabilized mature milk, we excluded the colostrum within one week of childbirth. Samples from mothers who developed disease or were administered antibiotics during lactation were also excluded.

\subsection{EVs isolation and DNA extraction from human milk samples}

To separate the EVs from HBM, EVs in milk samples were isolated using differential centrifugation at $10,000 \times \mathrm{g}$ for $10 \mathrm{~min}$ at $4{ }^{\circ} \mathrm{C}$. After centrifugation, the pellet contained bacteria and supernatant contained the EVs. Bacteria and foreign particles are thoroughly eliminated by sterilizing the supernatant through a $0.22 \mu \mathrm{m}$ filter. To extract the DNA from bacteria and bacterial EVs, bacteria and EVs were boiled for $40 \mathrm{~min}$ at $100{ }^{\circ} \mathrm{C}$. To eliminate the remaining floating particles and waste, the sample was centrifuged at $13,000 \mathrm{rpm}$ for $30 \mathrm{~min}$ at $4{ }^{\circ} \mathrm{C}$, and the supernatant was collected. DNA was extracted using a DNA isolation kit (DNeasy PowerSoil Kit, QIAGEN, Germany), in accordance with standard protocol provided along with the kit. The DNA from bacteria and EVs in each sample was quantified using the QIAxpert system (QIAGEN, Germany).

\subsection{Bacterial metagenomic analysis using DNA from human milk samples}

Bacterial genomic DNA was amplified with 16S_V3_F (5' - TCG GCA GCG TCA GAT GTG TAT AAG AGA CAG CCT ACG GGN GGC WGC AG -3') and 16S_V4_R (5' - GTC TCG TGG GCT CGG AGA TGT GTA TAA GAG ACA GGA CTA CHV GGG TAT CTA ATC C - $3^{\prime}$ ) primers, which are specific for the V3-V4 hypervariable regions of the $16 \mathrm{~S}$ rDNA gene. The libraries were prepared using PCR products according to MiSeq System guide (Illumina, USA) and quantified using QIAxpert (QIAGEN, Germany). Each amplicon was then quantified, set equimolar ratio, pooled, and sequenced on a MiSeq (Illumina, USA) according to the manufacturer's recommendations. 


\subsection{Analysis of bacterial composition in the microbiota}

Paired-end reads that matched the adapter sequences were trimmed by cutadapt version 1.1.6 [11]. The resulting FASTQ files containing paired-end reads were merged with CASPER and then quality filtered using a Phred (Q) score-based criteria described by Bokulich [12,13]. Any reads shorter than $350 \mathrm{bp}$ and longer than $550 \mathrm{bp}$ after merging were also discarded. To identify the chimeric sequences, a reference-based chimera detection step was performed using VSEARCH against the SILVA gold database [14,15]. Next, the sequence reads were clustered into Operational Taxonomic Units (OTUs) using VSEARCH with a de novo clustering algorithm under a threshold of $97 \%$ sequence similarity. The representative sequences of the OTUs were finally classified using GREENGENES database with UCLUST (parallel_assign_taxonomy_uclust.py script on QIIME version 1.9.1) under default parameters [16].

\subsection{Statistical analysis}

The richness (rarefaction curves) was analyzed using Chao1 and alpha-diversity indices (Chao1, ACE, Shannon index, Simpson index, and Fisher's index) were calculated. Significance between subgroups was tested using $t$-test for continuous variables. Principal component analysis (PCA) based on Euclidean distance was performed for quantifying the relationships between samples. In addition, networks were constructed using Pearson correlation coefficient. All statistical analyses were performed using $\mathrm{R}$ version 3.3.2.

\section{Results}

\subsection{Characteristics of the lactating mothers}

A total of 22 lactating mothers were enrolled in this study; the mean age was $33.1 \pm 3.4$ years. Of the 22 lactating mothers, $16(72.7 \%)$ were primiparous and $6(27.3 \%)$ were multiparous. Eight (36.4\%) mothers had vaginal delivery and 14 (63.6\%) mothers had delivered by Caesarian section (CS). Samples were collected on mean $11.1 \pm 2.4$ days after delivery.

\subsection{Diversity}

When analyzing Chao 1 and ACE to analyze the alpha diversity richness, bacteria showed significantly higher alpha diversity than bacterial EVs $(p<0.001$ and $p=0.005$, respectively). Bacteria showed higher Fisher's index values than the EVs, indicating higher evenness in the bacterial samples $(p<0.001)$. However, EVs and bacteria did not show any significant difference in Shannon diversity index $(p=0.51)$, which signifies richness, and Simpson's index $(p=0.05)$, which signifies evenness (Figure 1). The number of reads for bacteria, 14966.6 (SD 10189.1), was higher than that for EVs, 9267.8 (SD 6507.0). Bacteria also showed significantly higher levels of operational taxonomic units (OTUs), with 1202.3 (SD 789.1) reads compared to 501.0 (SD 446.8) reads in EVs.

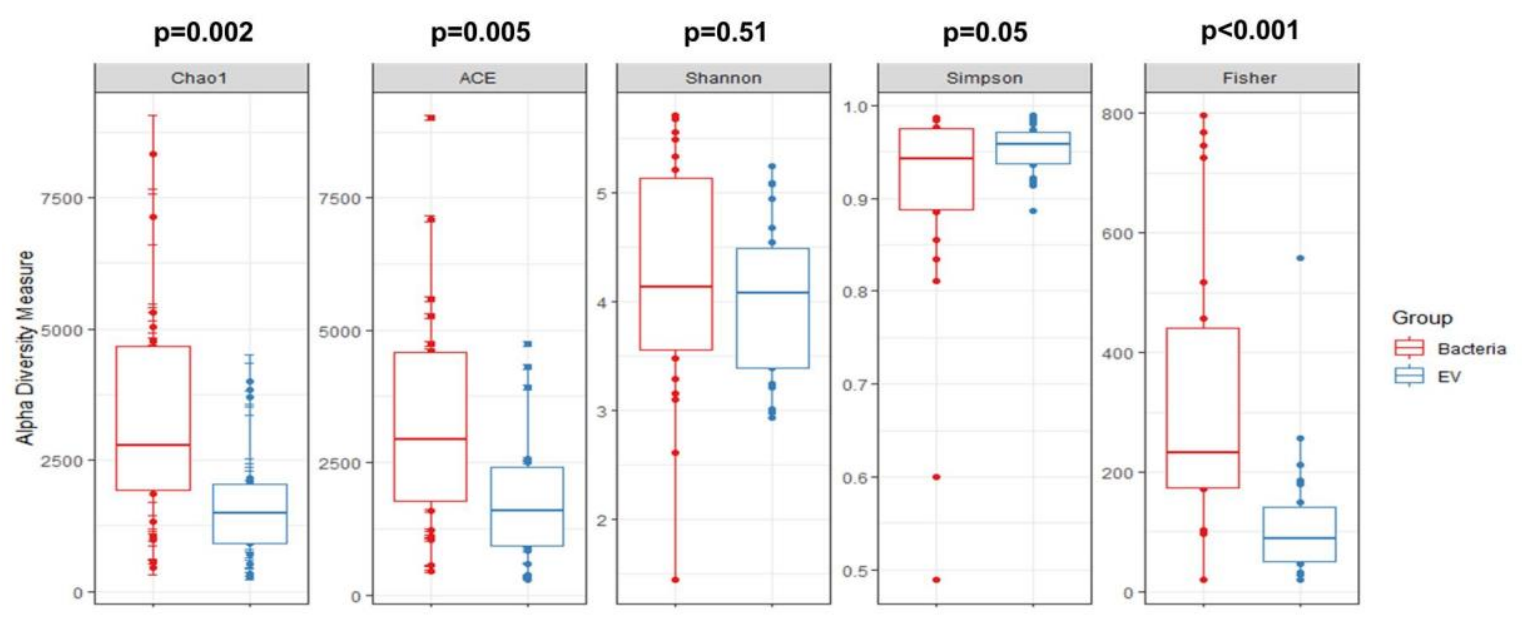


Figure 1. Alpha diversity analysis showed that microbial richness and evenness were significantly higher in bacteria samples than that in bacterial extracellular vesicle (EV) samples when analyzing using Chao 1 ( $p=0.002)$, ACE ( $p=0.005)$, and Fisher $(p<0.001)$. Bacteria and bacterial EV samples did not show any significant difference in Shannon diversity index $(\mathrm{p}=0.51)$ and Simpson's index $(p=$ 0.05).

The beta diversity of all samples was analyzed using PCA based on the Euclidean distance (Figure 2). The Euclidean distance value and cluster in the breast milk samples did not differ significantly between different individual samples at the EV level. However, the Euclidean distance values showed a significant difference in different individual samples at the bacteria level. Furthermore, the Euclidean distance of bacteria and EV samples from an identical sample showed a significant difference.

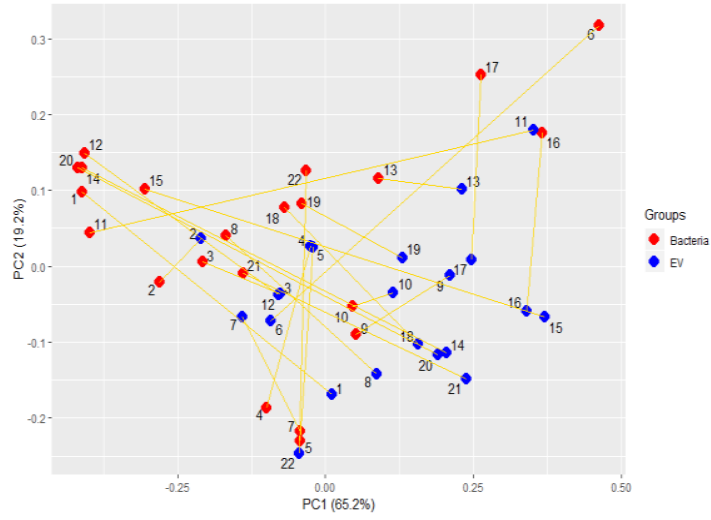

(a)

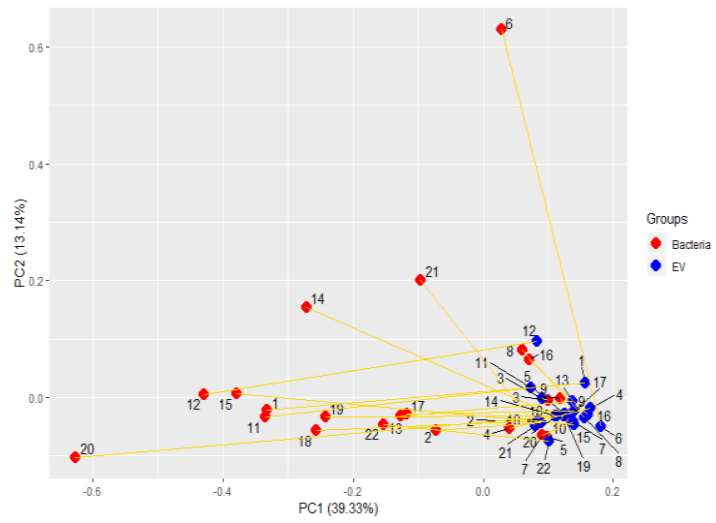

(b)

Figure 2. Beta diversity analysis using PCA based on the Euclidean distance (a) at phylum level and (b) at genus level.

\subsection{Bacterial composition}

We detected 23 phyla by analyzing the microbiota originating from breast milk. Among them, Firmicutes accounted for the largest proportion with 56.4\% (SD 19.4) among the phyla, followed by Proteobacteria (19.6\% (SD 19.1)), Bacteroides (9.8\% (SD10.5)), and Actinobacteria (9.0\% (SD7.9)). Verrucomicrobia showed an average proportion below $5 \%$, while other phyla accounted for less than $1 \%$ of the bacterial composition of breast milk (Figure 3a). Three hundred and ninety two genera were detected in total at the genus level, with Streptococcus accounting for the highest proportion at $25.1 \%$ (SD 20.9) and followed by Staphylococcus at $10.7 \%$ (SD 12.3). Bacteroides, Acinetobacter, Enterobacteriaceae(f), Ruminococcaceae(f), Bifidobacterium, Prevotella, Clostridiales(o), Corynebacterium, Akkermansia, Lactobacillus, Pseudomonas, Dialister, Stenotrophomonas, Blautia, Sphingomonas, Haemophilus, Neisseria, Lachnospiraceae(f), Rothia, and Faecalibacterium showed average proportions (below $5 \%$ ) while other genera showed less than $1 \%$ of bacterial sample composition on average (Figure $3 b$ ).

Meanwhile, bacterial EV analysis revealed a total of 22 phyla. Firmicutes accounted for the largest proportion $35.8 \%$ (SD 12.9), while Proteobacteria $24.5 \%$ (SD 13.3)), Bacteroidetes (15.5\% (SD 8.8)), and Actinobacteria (13.1\% (SD 8.3)) accounted for the remaining majority of the bacterial EV composition. Verrucomicrobia and Cyanobacteria accounted for less than $5 \%$ on average of the bacterial EV microbiome, and other phyla accounted for less than 1\% on average (Figure 3c). A total of 291 genera were detected at the genus level in bacterial EV samples. Bacteroides, Acinetobacter, and Lactobacillaceae(f) accounted for high proportions of EV samples with (9.1\% (SD 5.4), 6.9\% (SD 8.2), and 5.5\% (SD 9.1), respectively). Meanwhile, Streptococcus, Staphylococcus, Enterobacteriaceae(f), Ruminococcaceae(f), Bifidobacterium, Prevotella, Clostridiales(o), Corynebacterium, Akkermansia, Lactobacillus, Dialister, Stenotrophomonas, Blautia, Sphingomonas, Streptophyta(o), Comamonadaceae(f), 
Collinsella, Actinomyces, Clostridiaceae(f), and Burkholderia accounted for less than $5 \%$ of the bacterial EV composition on average. The rest of the detected genera accounted for less than $1 \%$ of the bacterial EV composition on average (Figure 3d).
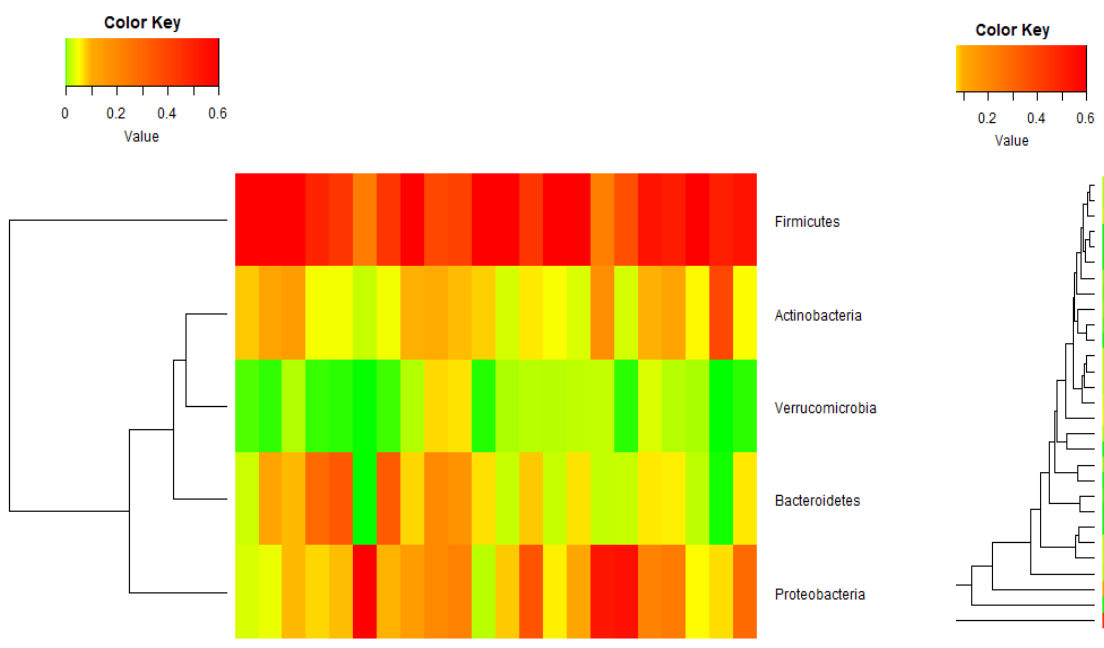

(a)

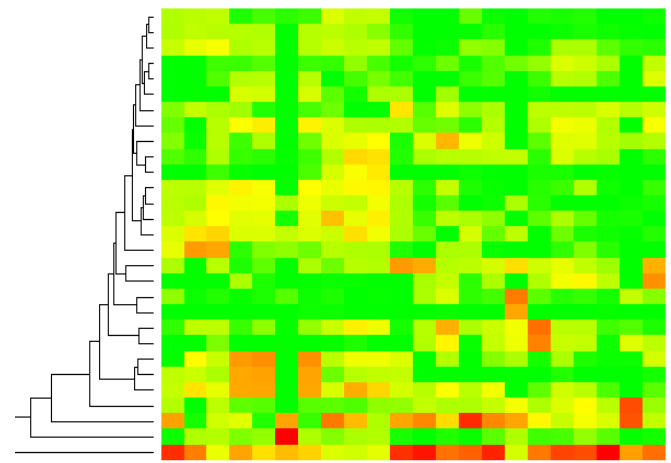

(b)
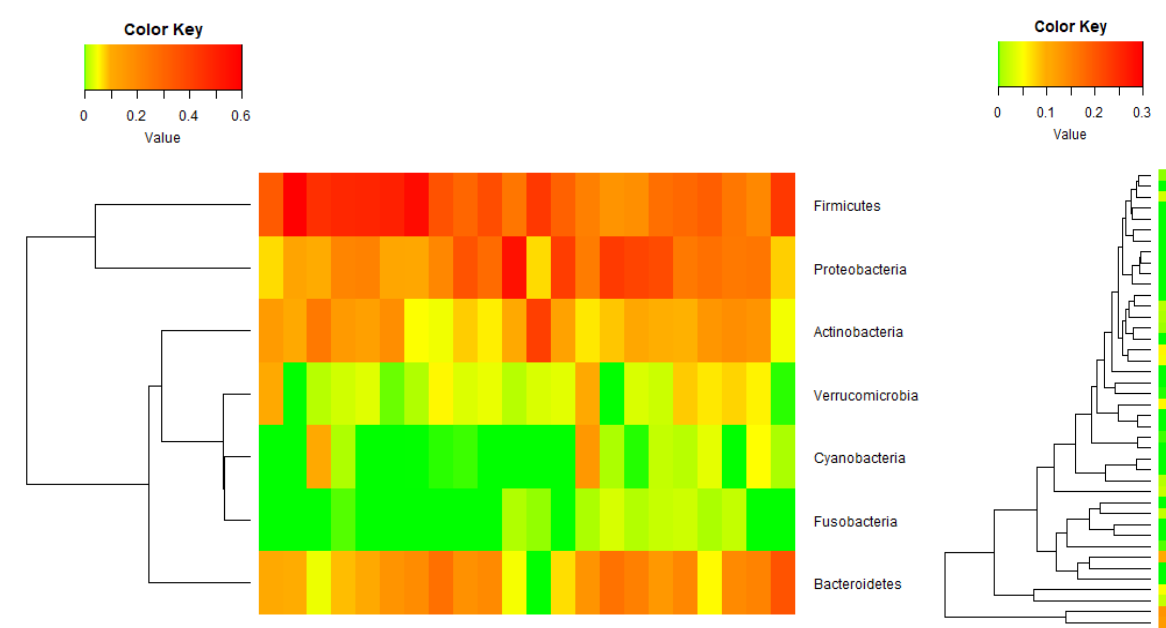

(c)

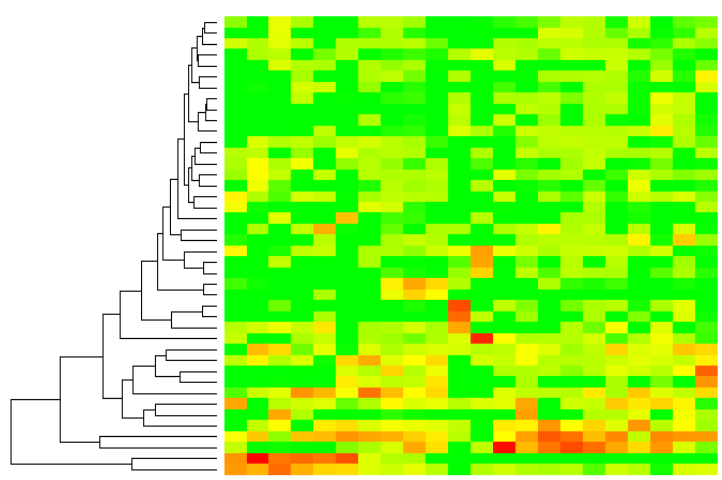

(d)

Figure 3. The composition of human breast milk microbiota in this study. (a) bacteria at phylum level, (b) bacteria at genus level, (c) bacterial extracellular vesicles (EVs) at phylum level, and (d) bacterial EVs at genus level.

\subsection{Correlation between bacteria and bacterial EVS}

We analyzed the correlation between bacteria isolated from HBM (bacteria) and bacterial EVs at the phylum level and determined that there were no taxa with significant correlation between the bacteria and bacterial EV samples. However, at the genus level we did determine significant proportional differences between several genera. We analyzed these correlation to determine any tendencies and found that Acinetobacter, Ruminococcaceae(f), Bifidobacterium, Comamonadaceae(f), Rothia, and Clostridiaceae(f) showed significant positive correlation between bacteria derived from HBM and bacterial EV samples ( $p<0.05$ ) (Figure 4, Table S1). 

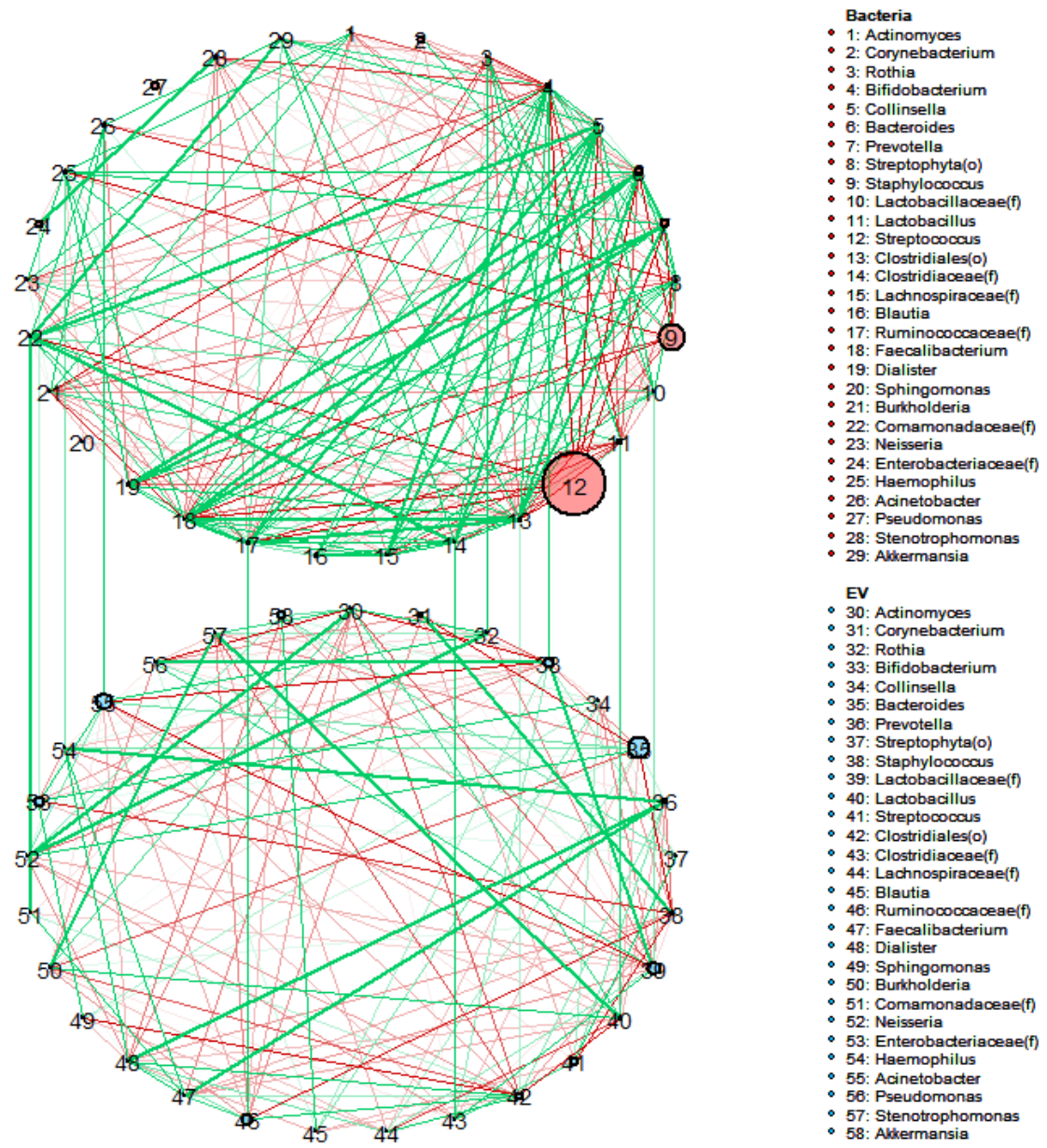

Figure 4. Correlation analysis between bacteria and bacterial extracellular vesicles samples showed a significant positive correlation for Acinetobacter, Ruminococcaceae(f), Bifidobacterium, Comamonadaceae(f), Rothia, and Clostridiaceae(f) $(\mathrm{p}<0.05)$. (see also Table S1)

Comamonadaceae(f) showed particularly high correlation between the two sample groups $(\mathrm{r}=$ 0.95). Although they account for less than $1 \%$ of the total proportion, Comamonas and Diaphorobacter also showed significantly high correlation between bacteria and bacterial EV samples $(r=0.94)$. Among the bacteria accounting for less than $1 \%$ of the total proportion, 24 genera showed significant positive correlation. Meanwhile, Bacteroides, Prevotella, Lachnospiraceae(f), Collinsella, and Burkholderia showed a trend of negative correlation, although this trend was not significant. Although some genera accounted for less than $1 \%$ average proportion with negative correlation, it was not significant.

\subsection{Difference between sub-groups}

We also analyzed the bacterial composition of HBM at the genus level based on parity. We determined 0.31- and 0.18-fold change between bacteria and bacterial EVs samples with respect to Staphylococcus and Collinsella, respectively, which is significantly lower when the breast milk is obtained from multiparous mothers. Meanwhile, the abundance of Haemophilus was shown to be 2.86-fold higher in HBM of multiparous mothers than in that of primiparous mothers $(p<0.05)$ (Figure 5a). No significant difference in the microbiome based on parity was observed in bacterial EV samples. When we compared the microbial composition of HBM of mothers who delivered by CS with those of mothers who had vaginal delivery, Streptophyta(o) was significantly higher (6.24-fold) in bacteria samples from mothers who delivered by CS (Figure 5b). The abundance of Actinomycetales was significantly lower $(0.19$-fold) in bacterial EV samples from mothers who 
delivered by CS (Figure 5b). A comparative analysis was conducted comparing calories, however no microbiome showed any significant difference.

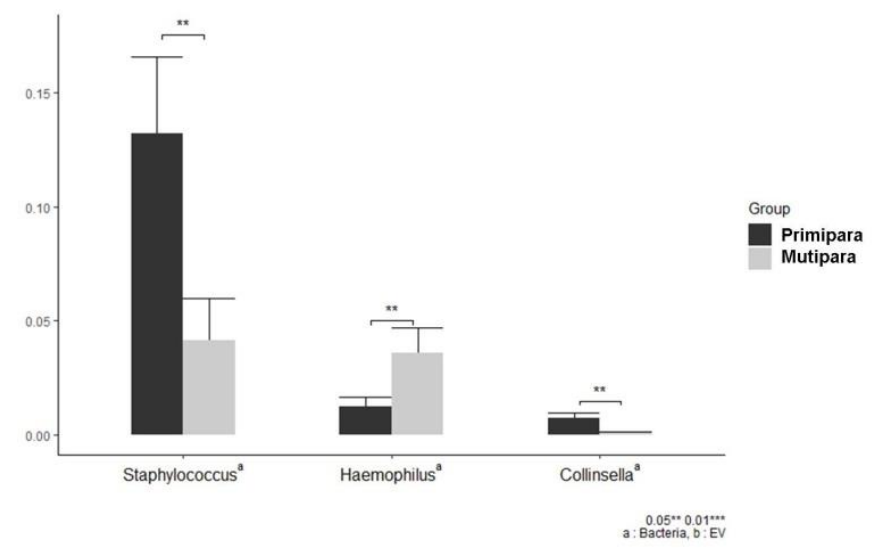

(a)

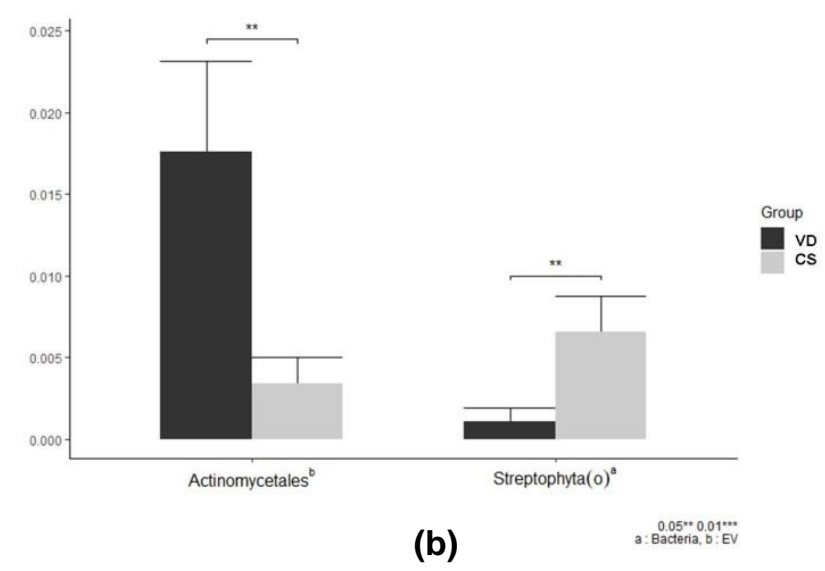

Figure 5. Subgroup analysis of bacterial composition of human breast milk based on (a) parity and (b) mode of delivery.

\section{Discussion}

To the best of our knowledge, this is the first study to analyze both microbiota and bacteria-derived EVs in HBM using next generation sequencing (NGS) in Korea. In the present study, we found that HBM of healthy lactating mothers contained diverse and complex bacterial communities, with Firmicutes as the predominant phyla. An analysis at the genus level showed significant proportional differences between microbiota and bacteria-derived EVs samples and several genera showed significant positive correlation between microbiota and bacterial EVs.

An initial study that analyzed the HBM using NGS suggested that there was a common core microbiome consisting of 9 bacterial genera [17]. Several subsequent studies also investigated which bacteria make up the core HBM microbiome. However, the results differed from study to study $[6,18,19]$. The core HBM microbiome has been the subject of controversy as it is thought to play a major role in the colonization of infant gut. A systematic review analyzed 242 studies and found that most of the bacterial species isolated from the breast tissue and milk belonged to four bacterial phyla, Firmicutes, Proteobacteria, Actinobacteria and Bacteroidetes [20]. A recent study, which reviewed 44 studies investigating 3105 breast milk samples from 2655 women, showed that the most frequently found genera were Staphylococcus, Streptococcus, Lactobacillus, Pseudomonas, Bifidobacterium, Corynebacterium, Enterococcus, Acinetobacter, Rothia, Cutibacterium, Veillonella, and Bacteroides [21]. Another systematic review of 12 studies using only culture independent method for healthy mothers reported that Staphylococcus and Streptococcus were the predominant genera, and 
that these genera may be universally present in HBM [5]. In our study, Firmicutes accounted for the largest proportion among the phyla, followed by Proteobacteria, Bacteroides, and Actinobacteria in both bacteria and bacterial EV samples. At the genus level, Streptococcus (25.1\%) and Staphylococcus $(10.7 \%)$ were predominant in bacteria samples. These results of the present study are in accordance with those of previous studies. However, analysis at the genus level showed significant proportional differences between bacteria and bacterial EVs.

As no research has been done to analyze bacteria-derived EVs in HBM, EVs released from the HBM microbiota are bound to be a part of the findings. A study has reported that intraperitoneally-injected bacterial EVs, spread throughout the mouse body, with accumulations in the liver, lung, spleen, and kidney within $3 \mathrm{~h}$ of administration [22]. Several studies have also reported that bacteria-derived EVs can be detected in human blood serum, urine or stool and claimed that bacteria-derived EVs are related to immune response or development of disease [23-27]. For example, studies have shown that EVs derived from Staphylococcus aureus contribute to atopic dermatitis like skin inflammation [28,29]. It is thought that not only pathogens, but also commensal bacteria can secrete EVs. One study revealed that commensal bacteria can produce EVs containing immunomodulatory molecules and affect the host immunity and health [30], while others suggested that EVs derived from commensal bacteria can be involved in gut colonization [31]. In the present study, we were able to detect bacterial EVs in HBM and unlike the dominant genera in bacteria sample, Bacteroides (9.1\%), Acinetobacter (6.9\%), and Lactobacillaceae(f) (5.5\%) accounted for a high proportion. When we analyzed the alpha diversity, the present study showed that the number of reads and OTUs in bacteria samples were higher than those in bacterial EVs. Bacteria samples also showed higher richness and evenness than bacterial EVs samples. Beta diversity analyses showed significant differences between bacteria and bacterial EVs within identical individual samples. The results of the present study indicate that while abundant bacterial communities are distributed evenly in HBM, the bacteria that release EVs do not necessarily match the bacteria present in HBM in high proportion and do not distribute evenly. Our findings suggest the presence of key bacteria with significant metabolic activity that are independent of the major bacterial populations that inhabit HBM.

The origins of bacteria in HBM are still unclear. It has been suggested that commensal bacteria from maternal skin (such as Staphylococcus, Corynebacterium, and Cutibacterium) or the infant's mouth (such as Streptococcus) can enter the mammary duct during breastfeeding [32]. Another hypothesis is that the maternal gut bacteria enter the mammary glands through the entero-mammary pathway, which would require the cells to penetrate the intestinal epithelium and reach the mammary glands through the bloodstream [33-35]. The observation that anaerobic bacteria such as Bacteroides, Bifidobacterium, Parabacteroides, and Clostridium that are not found on the skin are detected and shared between HBM and infant feces supports this hypothesis. In our study, gut-associated genera such as Bacteroides and Lactobacillaceae(f)) accounted for a high proportion of the genera within the bacterial EV sample. These findings thus suggest that the EVs derived from the gut bacteria may be involved in the vertical transfer of the gut microbiota from the mothers to infants.

Evaluation of the correlation between HBM-derived bacteria and bacterial EV samples revealed that several genera were significantly positively correlated. As a strong correlation can be interpreted as indicative of high metabolic activity of the bacteria, the effect of these bacteria on gut colonization is worth considering. We especially highlighted Bifidobacterium, as the gut microbiota of infant feces usually exhibits high abundance of this bacterium, with exclusively breastfeeding infants in particular exhibiting relatively abundant Bifidobacterium in the feces [36,37]. [35,36]. Several lines of evidence suggest that the relative level of Bifidobacterium abundance correlates with the development of disease or immunity. For example, a systematic review compared 101 studies on healthy controls with 147 studies on infected patients, all of which included molecular analysis of HBM. Some species were found only in infected patients whereas others were never detected in 
infected patients and were present only in healthy controls; specifically, Bifidobacterium and Lactobacillus were associated with the absence of infection [20]. In the present study, Lactobacillus was among the bacteria present at high abundance in bacterial EV samples and Bifidobacterium was identified as being highly correlated between bacteria and bacterial EVs. Together, these findings warrant additional research to explore the effects and functions of bacterial EVs on infant gut colonization and immune development.

Various factors such as mode of delivery, maternal weight, antibiotics, lactation stage, gestational age, geographical location, and maternal health have been found to contribute to the diversity of the HBM microbiota [1]. In the present study, subgroup analysis revealed that the abundance of Staphylococcus in the bacteria sample and Collinsella in the bacterial EV sample was significantly lower, and Haemophilus in bacteria sample was significantly higher when the HBM was obtained from multiparous compared to primiparous mothers. In contrast, a large study did not identify an effect of parity on the composition of the HBM microbiota [38]. [37]. Our study also showed that the abundance Streptophyta(o) was significantly higher in the bacteria sample and that of Actinomycetales was significantly lower in the bacterial EV sample in the CS than that in the vaginal delivery group. It has been suggested that labor is associated with the composition of HBM microbiota via increased intestinal permeability and by facilitating a greater degree of entero-mammary transfer of maternal gut bacteria [39]. Women who delivered by CS have been reported have a higher relative abundance of Proteobacteria and Carnobacteriaceae, albeit a lower relative abundance of Bifidobacterium, Leuconostocaceae, and Lactobacillus [1]. Alternatively, some studies have found no association between the composition of the HBM microbiota and delivery mode $[38,40]$. We therefore postulate that the diversity of bacterial communities is affected by a number of variables such as host immunity, environmental factors, and microbial factors, resulting in quantitative and qualitative differences

Our study has some limitations. First, the sample size was relatively small. Second, we did not analyze communities simultaneously from maternal and infant's mouth, skin, and stool, so the origins of HBM bacteria are unclear. Third, we also did not demonstrate the process by which HBM microbiota and their EVs involved in the colonization of infant gut. Considering that bacteria reaching the infant's gut can affect immunity, it is more important to know which bacteria have active biologic function than just describing the composition of microbiota in HBM. It is necessary to determine whether the EVs analysis can predict the key bacteria that play a major role in the colonization of infant gut and what factors influence the release of bacterial EVs in HBM. Studies are also needed to explain how bacterial EVs in HBM affect composition of infant gut microbiota and change the infant health outcomes. This will be the next task of our future study. Despite these limitations, the present study was the first to analyze bacterial EVs in HBM and will be the basis for further study by suggesting the possibility that bacterial EVs in HBM contribute to the colonization of infant gut.

In conclusion, the present study revealed the diverse bacterial communities in the HBM of healthy lactating mothers along with that of bacterial EVs and the relationship between these factors. Our findings suggest the existence of key bacteria with metabolic activity in HBM and the possibility that EVs derived from these bacteria are involved in the vertical transfer of gut microbiota from the mothers to their progeny. Future studies will be needed to confirm the role of bacterial EVs in the HBM in colonization of the infant gut and to ultimately promote colonization with beneficial key bacteria in HBM.

Supplementary Materials: The following are available online at www.mdpi.com/xxx/s1, Table S1: Correlation between bacteria and bacterial extracellular vesicles. 
Author Contributions: Conceptualization, S.Y.K. and D.Y.Y.; methodology, D.Y.Y.; validation, D.Y.Y.; formal analysis, S.Y.K. and D.Y.Y.; investigation, S.Y.K. and D.Y.Y; resources, S.Y.K. and D.Y.Y.; data curation, S.Y.K. and D.Y.Y.; writing-original draft preparation, S.Y.K. and D.Y.Y.; writing-review and editing, S.Y.K. and D.Y.Y.; supervision, D.Y.Y.; project administration, D.Y.Y. All authors have read and agreed to the published version of the manuscript.

Funding: This study was supported by the National Research Foundation of Korea (NRF) grant funded by the Korea government (Ministry of Science and ICT, No. NRF-2017R1C1B5018217).

Acknowledgments: The authors are grateful to the lactating mother and the Areumcheil Postnatal Care Center for their participation in this study and thank MD Health Care Inc. for helping analyze the data.

Conflicts of Interest: The authors declare no conflict of interest.

\section{References}

1. Ojo-Okunola, A.; Nicol, M.; du Toit, E. Human Breast Milk Bacteriome in Health and Disease. Nutrients 2018, 10, doi:10.3390/nu10111643.

2. Kim, S.Y.; Yi, D.Y. Components of human breast milk: from macronutrient to microbiome and microRNA. Clinical and experimental pediatrics 2020, 10.3345/cep.2020.00059, doi:10.3345/cep.2020.00059.

3. Martin, R.; Langa, S.; Reviriego, C.; Jiminez, E.; Marin, M.L.; Xaus, J.; Fernandez, L.; Rodriguez, J.M. Human milk is a source of lactic acid bacteria for the infant gut. J Pediatr 2003, 143, 754-758.

4. Heikkila, M.P.; Saris, P.E. Inhibition of Staphylococcus aureus by the commensal bacteria of human milk. J Appl Microbiol 2003, 95, 471-478.

5. Fitzstevens, J.L.; Smith, K.C.; Hagadorn, J.I.; Caimano, M.J.; Matson, A.P.; Brownell, E.A. Systematic Review of the Human Milk Microbiota. Nutr Clin Pract 2017, 32, 354-364.

6. Murphy, K.; Curley, D.; O'Callaghan, T.F.; O'Shea, C.A.; Dempsey, E.M.; O'Toole, P.W.; Ross, R.P.; Ryan, C.A.; Stanton, C. The Composition of Human Milk and Infant Faecal Microbiota Over the First Three Months of Life: A Pilot Study. Sci Rep 2017, 7, 40597, doi:10.1038/srep40597.

7. Brown, L.; Wolf, J.M.; Prados-Rosales, R.; Casadevall, A. Through the wall: extracellular vesicles in Gram-positive bacteria, mycobacteria and fungi. Nature reviews. Microbiology 2015, $13,620-630$.

8. O'Donoghue, E.J.; Krachler, A.M. Mechanisms of outer membrane vesicle entry into host cells. Cellular microbiology 2016, 18, 1508-1517, doi:10.1111/cmi.12655.

9. Perez-Cruz, C.; Delgado, L.; Lopez-Iglesias, C.; Mercade, E. Outer-inner membrane vesicles naturally secreted by gram-negative pathogenic bacteria. PLoS One 2015, 10, e0116896, doi:10.1371/journal.pone.0116896.

10. Kang, C.S.; Ban, M.; Choi, E.J.; Moon, H.G.; Jeon, J.S.; Kim, D.K.; Park, S.K.; Jeon, S.G.; Roh, T.Y.; Myung, S.J., et al. Extracellular vesicles derived from gut microbiota, especially Akkermansia muciniphila, protect the progression of dextran sulfate sodium-induced colitis. PLoS One 2013, 8, e76520, doi:10.1371/journal.pone.0076520.

11. Martin, M. Cutadapt removes adapter sequences from high-throughput sequencing reads. EMBnet. journal 2011, 17, pp. 10-12. 
12. Kwon, S.; Lee, B.; Yoon, S. CASPER: context-aware scheme for paired-end reads from high-throughput amplicon sequencing. BMC bioinformatics 2014, 15 Suppl 9, S10, doi:10.1186/1471-2105-15-s9-s10.

13. Bokulich, N.A.; Subramanian, S.; Faith, J.J.; Gevers, D.; Gordon, J.I.; Knight, R.; Mills, D.A.; Caporaso, J.G. Quality-filtering vastly improves diversity estimates from Illumina amplicon sequencing. Nature methods 2013, 10, 57-59, doi:10.1038/nmeth.2276.

14. Rognes, T.; Flouri, T.; Nichols, B.; Quince, C.; Mahe, F. VSEARCH: a versatile open source tool for metagenomics. PeerJ 2016, 4, e2584, doi:10.7717/peerj.2584.

15. Quast, C.; Pruesse, E.; Yilmaz, P.; Gerken, J.; Schweer, T.; Yarza, P.; Peplies, J.; Glockner, F.O. The SILVA ribosomal RNA gene database project: improved data processing and web-based tools. Nucleic acids research 2013, 41, D590-596, doi:10.1093/nar/gks1219.

16. Caporaso, J.G.; Kuczynski, J.; Stombaugh, J.; Bittinger, K.; Bushman, F.D.; Costello, E.K.; Fierer, N.; Pena, A.G.; Goodrich, J.K.; Gordon, J.I., et al. QIIME allows analysis of high-throughput community sequencing data. Nature methods 2010, 7, 335-336, doi:10.1038/nmeth.f.303.

17. Hunt, K.M.; Foster, J.A.; Forney, L.J.; Schutte, U.M.; Beck, D.L.; Abdo, Z.; Fox, L.K.; Williams, J.E.; McGuire, M.K.; McGuire, M.A. Characterization of the diversity and temporal stability of bacterial communities in human milk. PLoS One 2011, 6, e21313, doi:10.1371/journal.pone.0021313.

18. Jimenez, E.; de Andres, J.; Manrique, M.; Pareja-Tobes, P.; Tobes, R.; Martinez-Blanch, J.F.; Codoner, F.M.; Ramon, D.; Fernandez, L.; Rodriguez, J.M. Metagenomic Analysis of Milk of Healthy and Mastitis-Suffering Women. J Hum Lact 2015, 31, 406-415, doi:10.1177/0890334415585078.

19. Urbaniak, C.; Angelini, M.; Gloor, G.B.; Reid, G. Human milk microbiota profiles in relation to birthing method, gestation and infant gender. Microbiome 2016, 4, 1, doi:10.1186/s40168-015-0145-y.

20. Togo, A.; Dufour, J.C.; Lagier, J.C.; Dubourg, G.; Raoult, D.; Million, M. Repertoire of human breast and milk microbiota: a systematic review. Future Microbiol 2019, 14, 623-641, doi:10.2217/fmb-2018-0317.

21. Zimmermann, P.; Curtis, N. Breast milk microbiota: A complex microbiome with multiple impacts and conditioning factors. J Infect 2020, 10.1016/j.jinf.2020.01.023, doi:10.1016/j.jinf.2020.01.023.

22. Jang, S.C.; Kim, S.R.; Yoon, Y.J.; Park, K.S.; Kim, J.H.; Lee, J.; Kim, O.Y.; Choi, E.J.; Kim, D.K.; Choi, D.S., et al. In vivo kinetic biodistribution of nano-sized outer membrane vesicles derived from bacteria. Small 2015, 11, 456-461, doi:10.1002/smll.201401803.

23. Yoo, J.Y.; Rho, M.; You, Y.A.; Kwon, E.J.; Kim, M.H.; Kym, S.; Jee, Y.K.; Kim, Y.K.; Kim, Y.J. $16 \mathrm{~S}$ rRNA gene-based metagenomic analysis reveals differences in bacteria-derived extracellular vesicles in the urine of pregnant and non-pregnant women. Exp Mol Med 2016, 48, e208.

24. Choi, Y.; Park, H.; Park, H.-S.; Kim, Y.-K. Extracellular Vesicles, a Key Mediator to Link Environmental Microbiota to Airway Immunity. Allergy Asthma Immunol Res 2017, 9, 101-106. 
25. Lee, Y.; Park, J.-Y.; Lee, E.-H.; Yang, J.; Jeong, B.-R.; Kim, Y.-K.; Seoh, J.-Y.; Lee, S.; Han, P.-L.; Kim, E.-J. Rapid Assessment of Microbiota Changes in Individuals with Autism Spectrum Disorder Using Bacteria-derived Membrane Vesicles in Urine. Exp Neurobiol 2017, 26, 307-317.

26. Choi, Y.; Kwon, Y.; Kim, D.K.; Jeon, J.; Jang, S.C.; Wang, T.; Ban, M.; Kim, M.H.; Jeon, S.G.; Kim, M.S., et al. Gut microbe-derived extracellular vesicles induce insulin resistance, thereby impairing glucose metabolism in skeletal muscle. Sci Rep 2015, 5, 15878, doi:10.1038/srep15878.

27. Kim, M.H.; Rho, M.; Choi, J.P.; Choi, H.I.; Park, H.K.; Song, W.J.; Min, T.K.; Cho, S.H.; Cho, Y.J.; Kim, Y.K., et al. A Metagenomic Analysis Provides a Culture-Independent Pathogen Detection for Atopic Dermatitis. Allergy Asthma Immunol Res 2017, 9, 453-461, doi:10.4168/aair.2017.9.5.453.

28. Hong, S.W.; Kim, M.R.; Lee, E.Y.; Kim, J.H.; Kim, Y.S.; Jeon, S.G.; Yang, J.M.; Lee, B.J.; Pyun, B.Y.; Gho, Y.S., et al. Extracellular vesicles derived from Staphylococcus aureus induce atopic dermatitis-like skin inflammation. Allergy 2011, 66, 351-359, doi:10.1111/j.1398-9995.2010.02483.x.

29. Hong, S.W.; Choi, E.B.; Min, T.K.; Kim, J.H.; Kim, M.H.; Jeon, S.G.; Lee, B.J.; Gho, Y.S.; Jee, Y.K.; Pyun, B.Y., et al. An important role of alpha-hemolysin in extracellular vesicles on the development of atopic dermatitis induced by Staphylococcus aureus. PLoS One 2014, 9, e100499, doi:10.1371/journal.pone.0100499.

30. Shen, Y.; Giardino Torchia, M.L.; Lawson, G.W.; Karp, C.L.; Ashwell, J.D.; Mazmanian, S.K. Outer membrane vesicles of a human commensal mediate immune regulation and disease protection. Cell host $\mathcal{E}$ microbe 2012, 12, 509-520, doi:10.1016/j.chom.2012.08.004.

31. Bryant, W.A.; Stentz, R.; Le Gall, G.; Sternberg, M.J.E.; Carding, S.R.; Wilhelm, T. In Silico Analysis of the Small Molecule Content of Outer Membrane Vesicles Produced by Bacteroides thetaiotaomicron Indicates an Extensive Metabolic Link between Microbe and Host. Frontiers in microbiology 2017, 8, 2440, doi:10.3389/fmicb.2017.02440.

32. Ramsay, D.T.; Kent, J.C.; Owens, R.A.; Hartmann, P.E. Ultrasound imaging of milk ejection in the breast of lactating women. Pediatrics 2004, 113, 361-367, doi:10.1542/peds.113.2.361.

33. Jost, T.; Lacroix, C.; Braegger, C.P.; Rochat, F.; Chassard, C. Vertical mother-neonate transfer of maternal gut bacteria via breastfeeding. Environmental microbiology 2014, 16, 2891-2904, doi:10.1111/1462-2920.12238.

34. Perez, P.F.; Dore, J.; Leclerc, M.; Levenez, F.; Benyacoub, J.; Serrant, P.; Segura-Roggero, I.; Schiffrin, E.J.; Donnet-Hughes, A. Bacterial imprinting of the neonatal immune system: lessons from maternal cells? Pediatrics 2007, 119, e724-732, doi:10.1542/peds.2006-1649.

35. Kordy, K.; Gaufin, T.; Mwangi, M.; Li, F.; Cerini, C.; Lee, D.J.; Adisetiyo, H.; Woodward, C.; Pannaraj, P.S.; Tobin, N.H., et al. Contributions to human breast milk microbiome and enteromammary transfer of Bifidobacterium breve. PLoS One 2020, 15, e0219633, doi:10.1371/journal.pone.0219633.

36. Turroni, F.; Peano, C.; Pass, D.A.; Foroni, E.; Severgnini, M.; Claesson, M.J.; Kerr, C.; Hourihane, J.; Murray, D.; Fuligni, F., et al. Diversity of bifidobacteria within the infant gut microbiota. PLoS One 2012, 7, e36957, doi:10.1371/journal.pone.0036957. 
37. Zimmermann, P.; Curtis, N. Factors Influencing the Intestinal Microbiome During the First Year of Life. The Pediatric infectious disease journal 2018, 37, e315-e335, doi:10.1097/inf.0000000000002103.

38. Moossavi, S.; Sepehri, S.; Robertson, B.; Bode, L.; Goruk, S.; Field, C.J.; Lix, L.M.; de Souza, R.J.; Becker, A.B.; Mandhane, P.J., et al. Composition and Variation of the Human Milk Microbiota Are Influenced by Maternal and Early-Life Factors. Cell host E microbe 2019, 25, 324-335.e324, doi:10.1016/j.chom.2019.01.011.

39. Cabrera-Rubio, R.; Collado, M.C.; Laitinen, K.; Salminen, S.; Isolauri, E.; Mira, A. The human milk microbiome changes over lactation and is shaped by maternal weight and mode of delivery. The American Journal of Clinical Nutrition 2012, 96, 544-551, doi:10.3945/ajen.112.037382.

40. Williams, J.E.; Carrothers, J.M.; Lackey, K.A.; Beatty, N.F.; York, M.A.; Brooker, S.L.; Shafii, B.; Price, W.J.; Settles, M.L.; McGuire, M.A., et al. Human Milk Microbial Community Structure Is Relatively Stable and Related to Variations in Macronutrient and Micronutrient Intakes in Healthy Lactating Women. J Nutr 2017, 147, 1739-1748, doi:10.3945/jn.117.248864. 